CONF-821049--19

DE8 3008695

\title{
Experimental Determination of the Energy Dependence of Defect Production
}

\author{
K. L. Merkle and Wayne E. King \\ Argonne National Laboratory, Argonne, IL 60439
}

A. C. Baily, K. Haga and M. Meshii

Northwestern UnIversity, Evanston, IL 60201

\section{DISCLAIMER}

This report was prepared as an account of work sponsored by an agency of the United States Government. Neither the United States Government nor any agency thereof, nor any of their employees, makes any warranty, express or implied, or "sumes any legal liability or responsibility for the accuracy, completeness, or usefulness oi uy information, apparatus, product, or process disclosed, or represents that its use would not infringe privately owned rights. Reference herein to any specific commercial product, process, or service by trade name, trademark, manufacturer, or otherwise does not necessarily constitute or imply its endorsement, recommendation, or favoring by the United States Government or any agency thereof. The views and opinions of authors expressed herein do not necessarily state or reflect those of the United States Government or any agency thereof.

\footnotetext{
*Work supported by the National Science Foundation and the U.S. Department of Energy.
} 


\title{
Experimental Determination of the Eqergy Dependence of Defect Production
}

\author{
K. L. Merkle and Wayne E. King \\ Argonne National Laboratory, Argonue, IL 60439
}

A. C. Baily, K. Haga and M. Meshii

Northwestern University, Evanston, IL 60201

\begin{abstract}
A knowledge of primary damage production as a function of recoll energy is essential for predicting defect production in radiation environments of practical interest. The damage function $v(T), 1 . e .$, the number of Frenkel pairs as a function of recoll energy is determined for Cu from electron and ion damage-rate measurements. $V(T)$ shows a plateau at $v=0.54$ which extends up to $\sim 7 \times \mathrm{m}_{\mathrm{d}}^{\mathrm{min}}$. Therefore, simple damage models, such as the modified Kinchin-Pease expression, are inappropriate not only at high recoil energies where stimulated recombination in cascades reduces defect production, but also in the single displacement regime. As a consequence, no simple relation between $T_{d}^{m i n}$ and $T_{d}^{a v}$ is expected to exist. A procedure is suggested which uses anisotropy measurements in combination
\end{abstract}

\footnotetext{
*Work supported by the Nationa: Science Foundation and the U.S. Department of Energy.
} 
with polycrystal electron and inn irradiations to construct absolute damage functions in metals.

\section{Introduction}

In the present paper, we are concerned with the primary-damage production as a function of recoll energy. In defect production studies attention is generally limited to averaged characteristics of damage observed in a single grain of the material for an isotopic distribution of recoil directions or for damage in a non-textured polycrystal. Even for such an averaged situation many damage parameters are necessary for a complete description of the primary damage state. However, we shall consider here only the damage function, $V(T), 1, e .$, the average number of Frenkel pairs (FP) produced by a recoll of energy $T$. The damage function allows the prediction of $N_{F}$, the number of FP's produced for 1rradiations with monoenergetic particles of energy $E$

$$
N_{F}=N \phi \int_{T} K(E, T) v(T) d i
$$

or for irradiations with a distribulion of particle energies

$$
N_{E}=N \int_{E} \int_{T} \phi(E) K(E, T) v(T) d E d T
$$

or for the production of defects along the range of a particle 


$$
N_{F}=\int_{E} \int_{T} \frac{K(E, T)}{S(E)} v(T) d T d E
$$

where $N$ is the number of atoms, $K(E, T)$ is the differential scattering cross section, $S(E)$ the stopping power, $\phi$ and $\phi(E)$ are the dose and the dosespectrum, respectively.

The motivation for determining the damage function is therefore this predictive capability regarding the defect production in practically any irradiation field. In addition to this, studies of the damage function may lead to an understanding of the physical processes involved when energetic recoils interact with lattice atoms.

In the past, electrical resistivity measurements have provided a convenient tool for monitoring defect production in metals. In the following the application of this method to studies of the damage function shall be discussed.

\section{Danage Rate Measurenents}

The damage rate, $1 . e .$, the change in rasistivity $\Delta p$ per particle dose is assumed to be proportional to the change in concentration $C$ of FP's

$$
\frac{d \Delta \rho}{d \phi}=\rho_{F} \frac{d C}{d \phi}
$$

where $\rho_{F}$ is the Frenkel pair resistivity. The tocal cross section for FPproduction $\sigma_{\text {FP }}$ can then be determined from measurements of the damage rate in the undamaged material

$$
\sigma_{F P}=\frac{1}{\rho_{F}}\left(\frac{d \Delta \rho}{d \phi}\right)_{\Delta \rho=0}
$$


Generally, several problems and uncertainties are encountered in establishing absolute damage rates: (1) $\rho_{F}$ is not known with good accuracy. (2) When using thin folls, the measurements have to be corrected for the electrical size effect. (3) Extrapolation to $\Delta \rho=0$ requires a knowledge of the saturation behavior. (4) The effective $\rho_{F P}$ may be changed due to clustering of point defects. The latter may accur as a function of dose or when energet1c displacement cascades are produced. The corrections due to items 2 and 3 can generally be obtained with good confidence, while item 4 introduces an uncertainty which is very difficult to estimate for large clusters (N 2100 ), while sma11 clusters are thought to have a FP resistivity very close to the sum of the resistivity of 1solated defects. 1,2

A major point of concern 1s, however, the uncertainty in Item 1 , the FP resistivity, which has important consequences regarding the damage function behavio: at low energy.

\section{Danage Function Hear Threshold $(v \leq 1)$}

\subsection{Fundarentals}

From single crystal electron irradiation experiments a threstold energy surface $T_{d}\left(\Omega_{2}\right)$ can be derived $1 f$ the assumption is valid that a recoll in the direction $\Omega_{2}$ creates just one FP whenever the recoll energy exceeds $T_{d}\left(\Omega_{2}\right) \cdot 3,4$ The damage function $1 s$ then given by ${ }^{4,5}$

$$
v(T)=\int_{\Omega_{2}} H\left[T-T{ }_{d}\left(\Omega_{2}\right)\right] \frac{d \Omega_{2}}{4 \pi} \text { for } v \leq 1
$$


where $H[x]=0$ for $x<0$ and $H[x]=1$ for $x \geq 0$.

Generally Fp production is dominated by recoils close to the directions of close packed lattice rows which lead via replacement sequences to well separated interstitial-vacancy pairs. As a consequence, the minimum threshold energy $T_{d}^{m i n}$ is followed by a steep increase of $V(T)$ in a small range of $\mathrm{T}$. From threshold anisotropy studies on $\mathrm{Cu}$, by $\mathrm{King}$ et al., it has recently been found that production of defects in the remalning directions usually requires much higher energies. 5,6 Therefore, the damage function shows a plateau at $v<1$. The height of the plateau is determined by the fractional solid angle subtended by the "easy" directions and the extent of the plateau depends on the magnitude of the threshold energles in the "difficult" directions.

A plateau at $v<1$ is expected in any crystalline material whenever there is a clear energy gap between the easy and hard directions. It has been shown that single crystal experiments can establish particularly well the low energy regions of the threshold surface, while considerable uncertaincies exist regarding the detalls of the high energy regions. 4,6 In terms of the damage function this means that the onset of damage and the helght of the plateau can be very well established from the experimental threshold surface, but not the detalls of the transition to higher energies.

Damage rate measurements as a function of energy and direction in single crystals also provide a means for an independent determination of the FP resistivity. $3,5,6,7$ This is an extremely important aspect of single 
crystal experiments since a knowledge of $\rho_{F}$ is indispensable for deriving absolute defect production rates from resistivity measurements. Unfortunately, up to this date most anisotropy measurements and their analyses have not been of sufficient extent and accuracy to provide $\rho_{F}$ values that are as precise as one would like to have them for damaye function studies $(\sim \pm 10 \%)$.

Once the FP-resistivity is established, electron damage rate measurements on polycrystals car be used to derive the damage function. The FP production cross section for monoenergetic particles is given by

$$
\sigma_{F}(E)=\frac{1}{\rho_{F}} \frac{d \Delta \rho}{d \phi}=\int_{T} K(E, T) v(T) d T
$$

If the differential scattering cross section $K(E, T)$ is known, this integral equation can be solved numerically for $v(T)$ using an experimentally determined set of $\sigma_{F}(E)$ values. In electron irradiations $K(E, T)$ is given by the Mott cross section. $V(T)$ is usually constructed by using multistep and Iinear functions that are adjusted such that the standard deviation between calculated and measured damage rates are minimized. 8-11

A check on the consistency of the data and analysis can be made by comparing the damage function derived from single crystal data via Eq. (6) with the damage function derived from polycrystal data (Eq. (7). In a recent compilation by Jung ${ }^{11}$ quite poor agreement between single crystal results is indicated for the fcc metals $\mathrm{Cu}$ and $\mathrm{Al}$. $\mathrm{Pt}$, however, shows consistency between the poly- and single-crystal analysis. 


\subsection{Electron Danage Rate Measurements in $\mathrm{Cu}$}

In this section results from HVEM resistivity damage rate measurements on polycrystalline thin film specimens are reported and compared to single crystal results. ${ }^{4-6}$ The main aspects of the experimental procedures have been previously described. ${ }^{4}$ In order to avoid texture imposed by a crystalline substrate, polycrystal films were prepared by vacuum evaporation of $\mathrm{Cu}$ onto thin layers of carbon. Damage rate measurements as a function of specimen tilt confirmed the absence of texture effects in the present experiments.

There are two basically equivalent approaches to constructing a damage function from damage rate measurements on polycrystals. The first is to use the FP resistivity as determined in anisotropy measurements and to construct the damage function from polycrystal data alone using Eq. (7). The second is to derive the low energy part of the damage function $\mathrm{T}_{\text {min }} \leq \mathrm{T} \leq 2 \mathrm{~T}_{\text {min }}$ directly from the experimental threshold surface via $\mathrm{Eq}$. (6). Low energy electron defect production (DP) cross sections are then calculated from this part of the damage function. The $\rho_{F}$ value to be used for the polycrystalline data is determined by the best match to tne polycrystal electron DP cross sections at low energy. The latter procedure is used in the present paper since it makes use only of the topology of the threshold surface which is largely independent of systematic errors (such as introduced by size effect, dosimetry, specimen geometry factor, deviations from Mathiessen's rule etc.) that may be present when irradiations on different specimens are compared. 
Figure 1 shows the calculated cross sections as a function of electron energy based on the threshold function in Fig. 2 and a FP resistivity of $\rho_{F}=2.6 \times 10^{-4} \Omega \mathrm{cm}$. The latter is derived as discussed above through a match to the detalled threshold function below the plateau. A straight line is a good approximation to the real $v(T)$ in this range. The height of the plateau is at $\nu=0.54$, slightly changed from the single crystal value due to the use of a different $\rho_{F}$. The most striking feature of our damage function is the very great range of the plateau which extends up to $\sim 7 \times T_{m i n}$. The existence of such a plateau was indicated by the molecular dynamics calculations of KIng and Benedek. ${ }^{5}$ The plateau is followed by a step rise in $V$ which is determined by a fit to high energy electron DP data. 10 It should be noted that the continuation of the straight line fitted does not go through the origtn, as in the Kinchin-Pease model. A satisractory match could not be obtained if a straight line through the ortgin was chosen. A low energy proton trradiation in the stopped mode ${ }^{2}$ supports the surprisingly steep onset of DP in the multiple displacement regime.

Comparison between the polycrystal and the single crystal damage function shows good agreement at low energy, however, the extent of the plateau is quite different. This comes from the fact that the details of the high energy threshold reglons are poorly known from single crystai results. ${ }^{4,6}$ Therefore, the transition from the placeau to $v=\Delta$ and beyond is best studied using polycrystal data. The single crystal analysis is 
important for establishing the value of $\rho_{F}$ and the details of the damage function near threshold.

\section{Hultiple Defect Production}

The approach of determining $v(\mathrm{~T})$ via Eq. (7) together with damage cross section measurements can be extended to much higher recoil energies by a suitable choice of irradiating particle mass and energy. Since the evaluation of damage production at high energy always had to rely heavily on theoretical concepts and since the DP experiments almost always are discussed in reference to simple theoretical models we shall interject at this point some remarks about the most widely used one.

\subsection{The Modified Kinchin-Pease Model}

The damage function in the modified Kinchin-Pease model ${ }^{12}$ is given by

$$
\begin{aligned}
0 \quad \text { for } T<T_{d}^{\text {eff }} \\
v_{K P}(T)=1 \quad \text { for } T_{d}<T<2.5 T_{d}^{\text {eff }} \\
\frac{k \varepsilon}{2 T_{d}^{e f f}} \text { for } \varepsilon \geq 2.5 T_{d}^{\text {eff }}
\end{aligned}
$$

where $K$ is the displacement efficiency factor $(K \neq 0.8), 13$ the damage energy $\varepsilon$ is the recoil energy reduced by the total electronic losses and $T_{d}^{e f f}$ is an effective threshold energy. A knowledge of the detailed threshold function $v(T)$ for $\nu \leq 1$ could now be used to improve on Eq. (8) by setting 


$$
\begin{aligned}
& v(T) \quad, v \leq 1 \\
& v_{L}(T)= \\
& \frac{k \varepsilon}{2 T_{d}^{e f f}}, \nu>1
\end{aligned}
$$

$v(T)$ for $v \leq 1$ would also be expected to serve as a guide for determining the effective threshold energy. If $0<v(T)<1$ is replaced by a step function and if $\nu\left(T_{1}\right)=1, T \frac{T^{e f f}}{\text { can be defined as }}$

$$
\int_{T_{d}^{\min }}^{T_{1}} \frac{d \sigma}{d T} v(T) d T=\int_{T_{d}^{e f f}}^{T} \frac{d \sigma}{d T} d T
$$

For hard sphere scattering $\mathrm{T}_{d}^{\text {eff }}=\mathrm{T}_{\mathrm{d}}^{\mathrm{av}}$, i.e., $\int_{\mathrm{T}_{\mathrm{min}}}^{\mathrm{T}} \nu(\mathrm{T}) \mathrm{dT}=\mathrm{T}_{1}-\mathrm{T}_{\mathrm{d}}^{\text {eff }}$. For real potentials $\mathrm{T}_{d}^{\text {eff }}<\mathrm{T}_{d}^{a v}$. Therefore, if $\mathrm{T}_{d}^{\text {eff }}$ in Eq. (9) is replaced by $T_{d}^{\text {av }}$, the displacement efficiency factor $k$ would be increased. However, sinze the correction factor depends on the shape of the threshold function no universal constant can be given.

The basic assumptions of the Kinchin-Pease model are strongly violated whenever $T_{1} \gg T_{d}^{m i n}$, as is Indicated by our experiments in Cu. Under these circumstances multiple defect production becomes much more complicated than implied by the simple model. Some of the consequences of anisutropy have been discussed in detail by Lucasson. ${ }^{14}$ Results from molecular dynamics calculations suggest that a linear approximation may be used in $\mathrm{Cu}$ for 
$1 \leq v(T)<2.5$ However, it should be pointed out that for $T_{1} \gg T_{d}^{m i n}$ the slope of the linear region in Eqs. (8) and (9) is not necessarily determined by $\mathrm{T}_{\mathrm{d}}^{\text {eff }}$ or $\mathrm{T}_{\mathrm{d}}^{\mathrm{av}}$ as calculated from Eq. (10). Empirically when the damage function is assumed to be linear in $E$ in this range $T_{d}^{\text {eff }}$ would then have to be determined from high energy electron or low energy proton damage rate measurements via Eqs. (7) and (9).

When comparing damage rate data with calculations of DP it is often convenient to use an efficiency factor

$$
\xi=\frac{N_{e}}{N_{t}}
$$

where $\mathrm{N}_{e}$ is the experimental and $\mathrm{N}_{t}$ the theoretical number of defects.

\subsection{Ion Irradiations}

Compared to electron irradiation, the range of available recoll energles is greatly increased in ton damage studies. The damage function can thus be studied over the whole range of interest in the multiple displacement and cascade regime. Two approaches have been used.

In the first approach thin target foils are irradiated with monoenergetic ion beams in transmission. The FP production cross section $\sigma_{F}$ is measured as a function of Ion mass and energy. Similar to the electron case, information on $v(T)$ can then be extracted from Eqs. (7) or (3) as long as the differential scattering cross sections are known. It is desirable to stay in energy well below the Coulomb barrier in order to 
avoid complications in the recoil spectrum due to nuclear reactions. 15 The differential scattering cross sections are generally obtained through the: formulation of Lindhard et al., 21 which is based on the Thomas-Fermi potential. In some instances improved potentials and more accurate cross sections are available. 22,23

In utflizing Eq. ( 7 ) it is assumed that the defects created by different recoils along the path of the incident ion are additive, that is that overlap effects are insignificant. This assumption s'ould be well fulfilled In light ion frradiations, but for heavy ion irradiations at low energy, damage overlap may be Important.

Figure 3 shows the fraction $F(T)$ of defects produced by recoils $\leq T$ for a selection of ion irradiations of $\mathrm{Cu}$.

$$
F(T)=\sigma_{F}^{-1} \int_{0}^{T} K(E, T) v(T) d T
$$

Recoil energies can be changed over a wide range, but damage in any single Irradiation is generally also produced over a wide distribution of recoll energies. This limits the sensitivity with which the damage function can be probed. A characteristic median energy $\mathrm{T}_{1 / 2}$, defined as $\mathrm{F}\left(\mathrm{T}_{1 / 2}\right)=0.5$ is useful in pointing out the magnitude of the recoll energies near which a majority of the damage is produced in any given irradiation. From Fig. 3 it is seen that $\mathrm{T}_{1 / 2}$ can range over several orders of magnitude. In order to have simflar recoll energies as in even the most energetic electron irradiations that have been used in the past in DP studies one has to 
Irradiate with protons at quite low energy. This necessitates very thin specimens, on the order of $100 \mathrm{~nm}$, If the recoll range near the threshold for multiple displacement is to be investigated.

In the second approach to studies of the damage function at high energy the irradiating particle is stopped inside the thin film specimen. In this manner the total number of defects produced along the path of the Ion can be measured. Particulariy for self-lons, the cascade initiated by the incoming ion (energy E) can be considered equal to a recoil cascade of energy $T=E$ whose damage function value $V(T)$ can thus be directly measured. 24 By irradiation with a number of different heavy Ions it has been found that the defect production at high energy $\left(\mathrm{T}_{1 / 2}>10 \mathrm{keV}\right)$ is proportional to the damage energy. 2 Such behavior is indeed expected in the subcascade regime. 26

\subsection{Danage Function of Cu at High Energy}

It would be most desirable to be able to construct a damage function based on electron and ion damage measurements on the same specimen. Such measurements will soot be possible at the ANL HVEM Tandem Facility. At present, however, there is a paucity of ion damage rate data on $\mathrm{Cu}$, in the important transition region between high energy electron and low enerby proton irradiations. The complete damage function In FIg. 4 is a continuation of the function in Fig. 2 to higher energies, using lon damage rates together with damage calculations using PINT0. ${ }^{2}$ At very high energy $\left(\mathrm{T}_{1 / 2}>10 \mathrm{keV}\right)$ it tas previously been found by Averback et al. ${ }^{2}$ that the 
number of defects is proportional to the damage energy. The slope of the damage function $V(E)$ in this region ( $4.2 \mathrm{FP} / \mathrm{keV})$ was obtained from the number of defects/cascade produced in self-ion bombardment, 24 taken as the average over the two avallable measurements at 500 and $560 \mathrm{keV} .2,24 \mathrm{~A}$ proton transmission value at low energy $(150 \mathrm{keV})^{2}$ was used for establishing the onset of the departure from the steep slope in the multiple displacement regime. An additional linear portion was used together with a smooth transition to approach the final slope of the damage function.

The goodness of fit of this damage function can be judged from a plct of the damage efficiency as a function of $T_{1 / 2}$. In FIg. 5 , results from high energy light Ion damage experiments 17,18 as wel1 as stopped lon data up to $0^{+}$and the self ion points are included. It is seen that the calculated damage rates according to our damage flinction agree very we11 with the experimental ones over the whole range. In contrast to this the efficlency strongly decreases with energy when the modified KInchin-Pease model is employed.

\section{Sumary and Discussion}

A recently derived threshold energy surface has indicated that the anisotropy In copper is more pronounced and that FP production is somewhat lower than had previously been thought. 5,6 The new FP resistivity determined in this work was used to obtaln polycrystal defect production crose sections from electron and ion irradiations. A damage function consistent wh the avallable data has been derived. This function 
exhibits an extended plateau at $\nu=0.54$ followed by a steep $r^{t}$ se up to $v \sim 4$. The transition to the final section in $v(T)$ which was assumed to be proportional to damage energy takes place in the range $300 \mathrm{eV}$ to $4 \mathrm{keV}$.

This transition to lower defect production in the keV range has previously been suggested to come to a large part from the stimulated recombination of $\mathrm{FP}^{\prime} \mathrm{s}$ during the cooling phase of energet/c cascades. Molecular dynamics simulations have shown that stimulated recombination of $\mathrm{FP}^{\prime} \mathrm{s}$ is a natural component of defect production also at low energy. 19 The damage function studies seem to suggest that appreciable enhanced recombination extends from seyeral hundred to several thousands of eV. At high energy che FP production is again proportional to $\varepsilon$ as a corsequence of subcascade formation.

If the damage function would increase from 0 to 1 in a rather narrow range of $T$, a step function approximation would be appropriate. The presence of an extended plateau at $v$ much below 1 , however, makes it Impossible to use a simple sharp-threshold model such as KInchIn-Pease for describing defect production.

The fractional solid angle subtended by the easy directions determines the helght of the plateau, which could therefore be expected to Increase from the light to the heavy metals. This would be consistent with a corresponding decrease in the ratio of the effective to the minimur chreshold energy as implied by Jung's analysis. 11

Since multiple defect production must be quite complicated in the presence of strong anisotropy ${ }^{14}$ it is not clear whether the continuation of 
the damage function in $1<v<4$ can be reasonably well approximated by a IInear function. Also, the steep rise in the damage function in this range is of considerable interest to our understanding of multiple defect production. However, any fine detail of the damage function in regions not close to threshold must be viewed with considerable caution because of inberent inaccuracies in the analysis. It would, however, be of interest to perform a detailed study of this range using a combination of high energy electron and low energy proton irradiation. A global analysis of highly accurate cata should certainly give a more detalled damage function In this important low energy range. Nevertheless, the detalled functional dependence may not be completely accessible experimentally.

The most important result of the present work is the existence of an extended plateau in $\mathrm{Cu}$ at $v<1$. This has interesting Implications concerning defect production mechanisms which will be discussed elsewhere.

In the context of damage prediction this means, however, that simple models such as KInchin-Pease are not only defictent in the cascade region but are also completely inadequate from a conceptual point of view whenever the threshold surface predicts strong anisotropy and an appreclable energy gap exists between easy and difficult directions.

Previous conclusions $2,16,19$ regarding the reduction of DP in cascades by stimulated recombination are relnforced by the present analysis. It is interesting to note that the transition starts out at quite low energy and 
that the spike effect is quite well developed al ready in cascades which produce less than 10 F''s.

We would like to suggest the following procedure for obtaining experimental information on the damage function:

1. Obtain threshold surface and FP resistivity by single crystal electron irradiation.

2. Establish damage function in low energy range by electron and proton Irradiation of polycrystals.

3. Determine defect production in self-ion or heavy-ion bombardment in the stopped mode to obtain the defect production in energetic cascades.

4. Use trial and error or minimization procedure to arrive at a damage function consistent with all defect production measurements.

\section{Acknowledgewents}

The authors are grateful to Dr. R. Benedek for valuable discussions and for supplying the PINTO program. We would also like to thank Dr. 0. S. Oen for providing the Mott to Rutherford cross section ratio up to $3 \mathrm{MeV}$. This work has been supported by the Metallurgy Program, Metallurgy and Materials Section, Division of Materials Research, National Solence Foundation, Washington, D.C. under Grant DMR-8114845 and by the U.S. Department of Energy. 


\section{References}

1. J. W. Martin, J. Phys. F $\underline{2}, 842$ (1972).

2. R. S. Averback, R. Benedek and K. L. Merkle, Phys. Rev. B18, 4156 (1978).

3. P. Jung, R. L. Chaplin, H. J. Fenz1, K. Reichelt and P. Wombacher, Phys. Rev. B $\underline{8}, 553$ (1973).

4. W. E. King, K. L. Merkle and M. Meshii, Phys. Rev. B23, 6319 (1981).

5. W. E. King, R. Benedek, K. L. Merkle and M. Mesh1i, Proceedings of the Yamada Conference $V$ on Point Defects and Defect Interacsions in Metals, Kyoto, 1981.

6. W. E. King, K. L. Merkle and M. Mesh11, these proceedings.

7. W. Schilling, J. Nuc1. Mat. $69 \& 70,465$ (1978).

3. A. Sosin and W. Bauer in Studies in Radiation Effects in Solids, G. J. Dienes, ed. (Gordon and Breach, 1969), p. 153.

9. G. W. Iseler, H. I. Dawson, A. S. Mehner and J. W. Kauffman, Phys. Rev. 146,468 (1966).

10. J. Wurm, KFA Jülich Report-581/FN (1969).

11. P. Jung, Phys. Rev. B23, 664 (1981).

12. M. J. Norgett, M. T. Robinson and I. M. Torrens, Nucl. Eng. Des. 33 , 50 (1975).

13. P. Sigmund, Radia. Eff. 1, 15 (1969).

14. P. Lucasson and A. Lucasson, Radia. Eff. 39, 195 (1978).

15. H. H. Andersen and H. Sørensen, Radis. Eff. 1449 (1972). 
16. K. L. Merkle, R. S. Averback and R. Benedek, Phys. Rev. Letters 38, $424(1977)$.

17. H. Saka1r1, E. Yagi, A. Koyama, T. Karasawa and R. liasiguti, J. Phys. Soc. Japan 43, 999 (1977).

18. B. R. Nielsen, Thesis, University of Aahus (1979).

19. R. S. Averback, R. Benedek, K. L. Merkle, J. Sprinkle and L. J. Thompson, J. Nucl. Mat., in print.

20. P. Jung, B. R. Nielsen, H. H. Andersen, J. F. Bak, H. Knudesn, R. R. Coltman, Jr., C. E. Klabunde, J. M. Williams, M. W. Guinan and C. E. Violet, In Effects of Radiation on Materials, H. R. Brager and J. S. Perrin, eds., ASTM 1982.

21. Y. Lindhard, V. Nielsen, M. Scharff and P. V. Thomsen, K\$il. Dan. Vidensk. Selsk. Mat. Fys. Medd. 33 No. 10 (1963).

22. W. D. Wilson, L. G. Haggmark, and J. P. Biersack, Phys. Rev. B15, 2458 (1977).

23. N. H. Sabel11, R. Benedek and T. L. G11bert, Phys. Rev. A20, 677 (1979).

24. W. Kesternich and K. L. Merkle, in Applications of Ion Beams to Metals, S. T. Picraux, E. P. EerNisse and F. L. Vook, eds. (Plenum, 1974), p. 495.

25. W. E. King and R. Benedek, Phys. Rev. B23, 6335 (1981).

26. K. L. Merkle, in Radiation Damage in Metals, N. L. Petersion and S. D. Hartnen, eds. (ASM 1976), p. 58. 
27. F. Dworschak, J. Nethäuser, H. Schuster, J. Wurm, S. Potyka, G. Sokolowsk1, and H. Wollenberger, Phys. Rev. 16, 685 (1966).

28. 0. S. Oen, private communication and in Cross Sections for Atomic Displacement in Solids by Fast Electrons, ORNL Report No. 4897 (1973).

29. W. E. King and R. Benedek, these proceedings. 


\section{PIgure Captions}

Fig. 1. Frenkel pair production cross sections for polycrystals at electron energles up to $3 \mathrm{MeV} . \Delta$ Ref. 10, + Ref. $27, \quad$ this work. The solid line ts the calculated cross section using the present damage function and the Mott-cross section. 28

Fig. 2. Damage function $v(T)$ near threshold. The dotted line indicates the threshold function obtained from the single-crystal analysis. ${ }^{6}$

Fig. 3. Fraction of Frenkel pairs produced in Cu by recolls below $T$ for electron and ion irradiations (transmission case).

Fig. 4. Damage function in Cu.

Fig. 5. Ratio of experimental to calculated defect production for various ions as a function of $\mathrm{T}_{1 / 2}$. The calculations are based on the present threshold funtion and the modified KInchin-Pease expression with $\mathrm{T}_{\mathrm{d}}^{\mathrm{av}}=29 \mathrm{eV}$. 


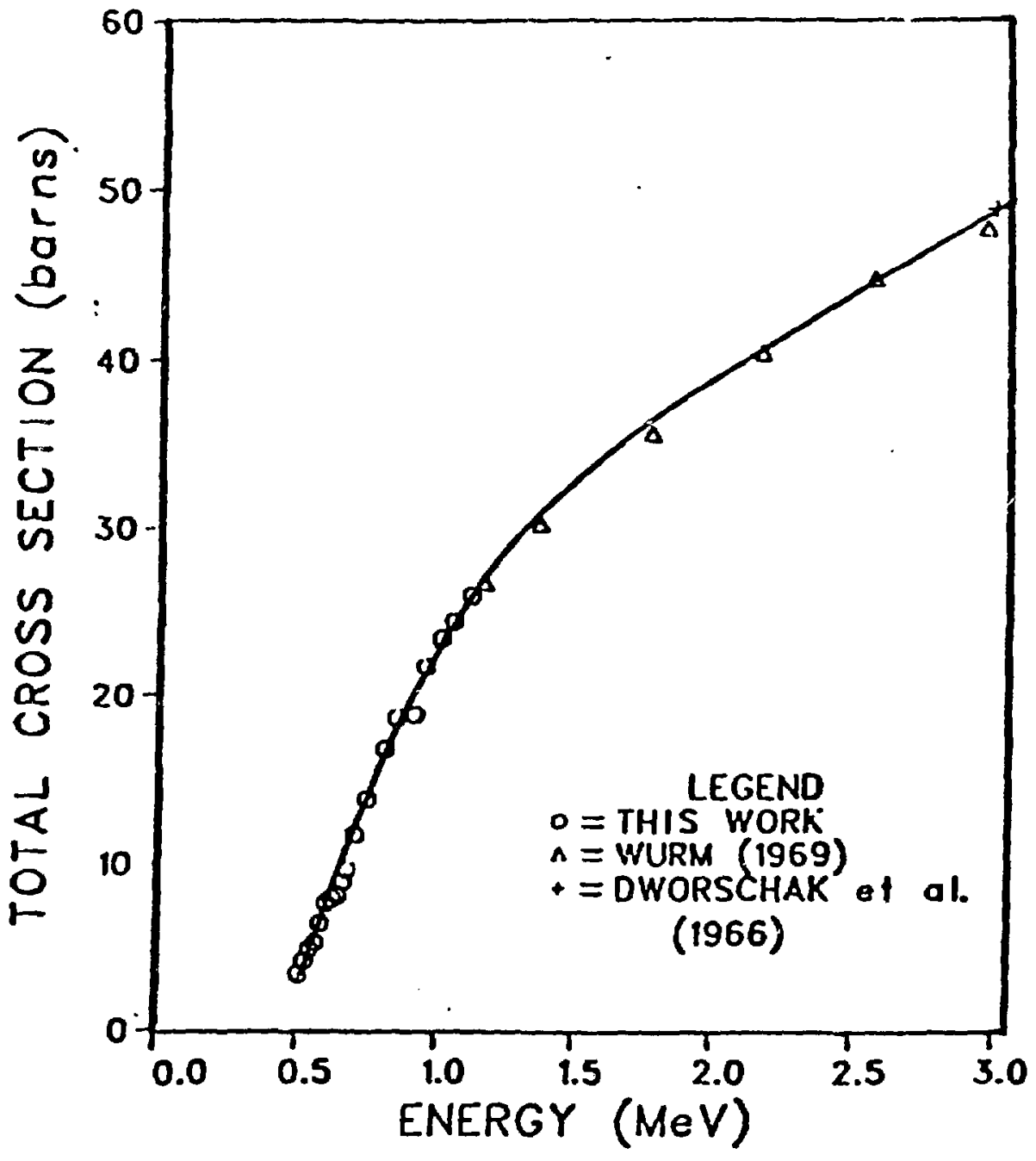

Fig. 1 


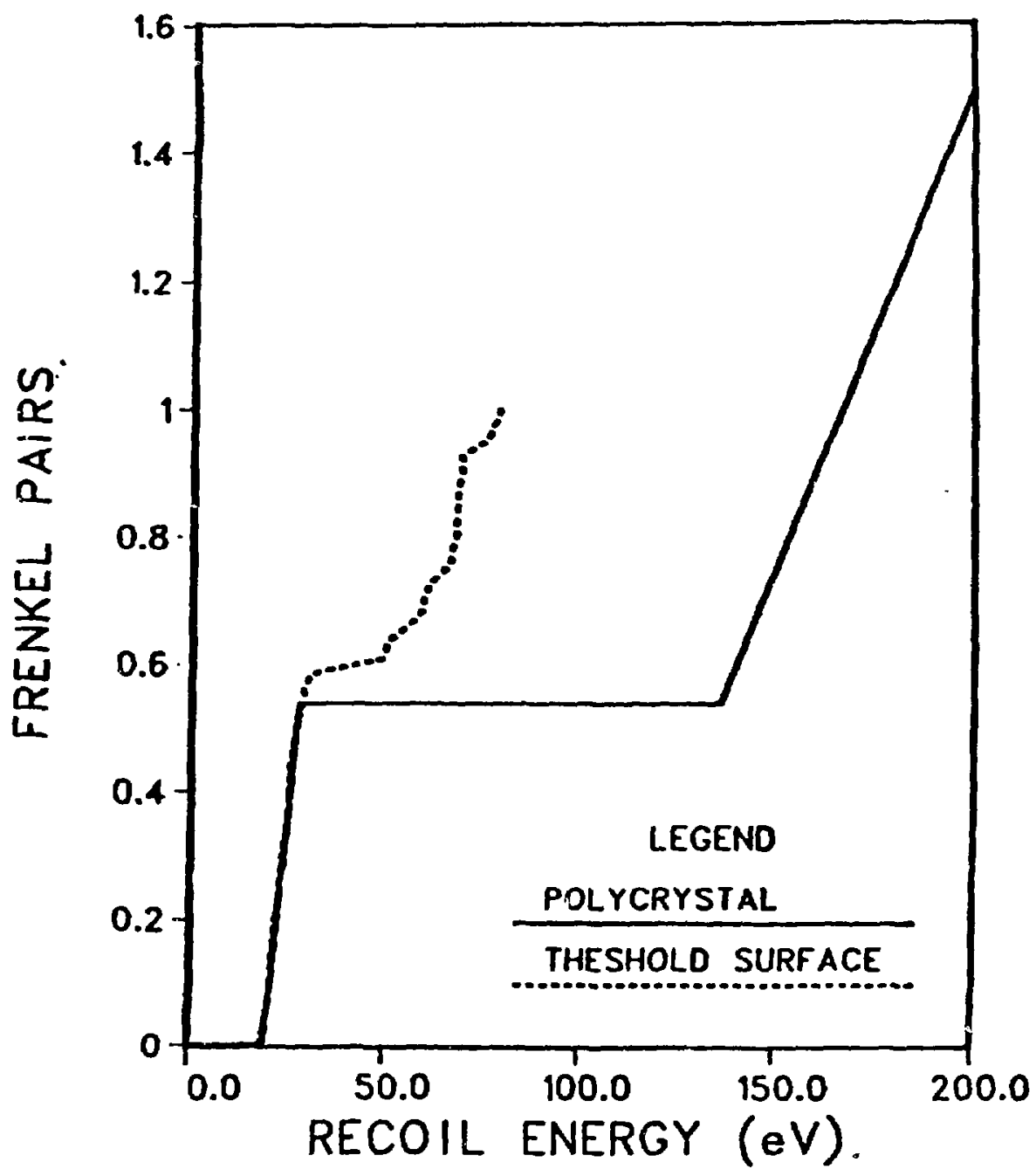

Fig. 2 


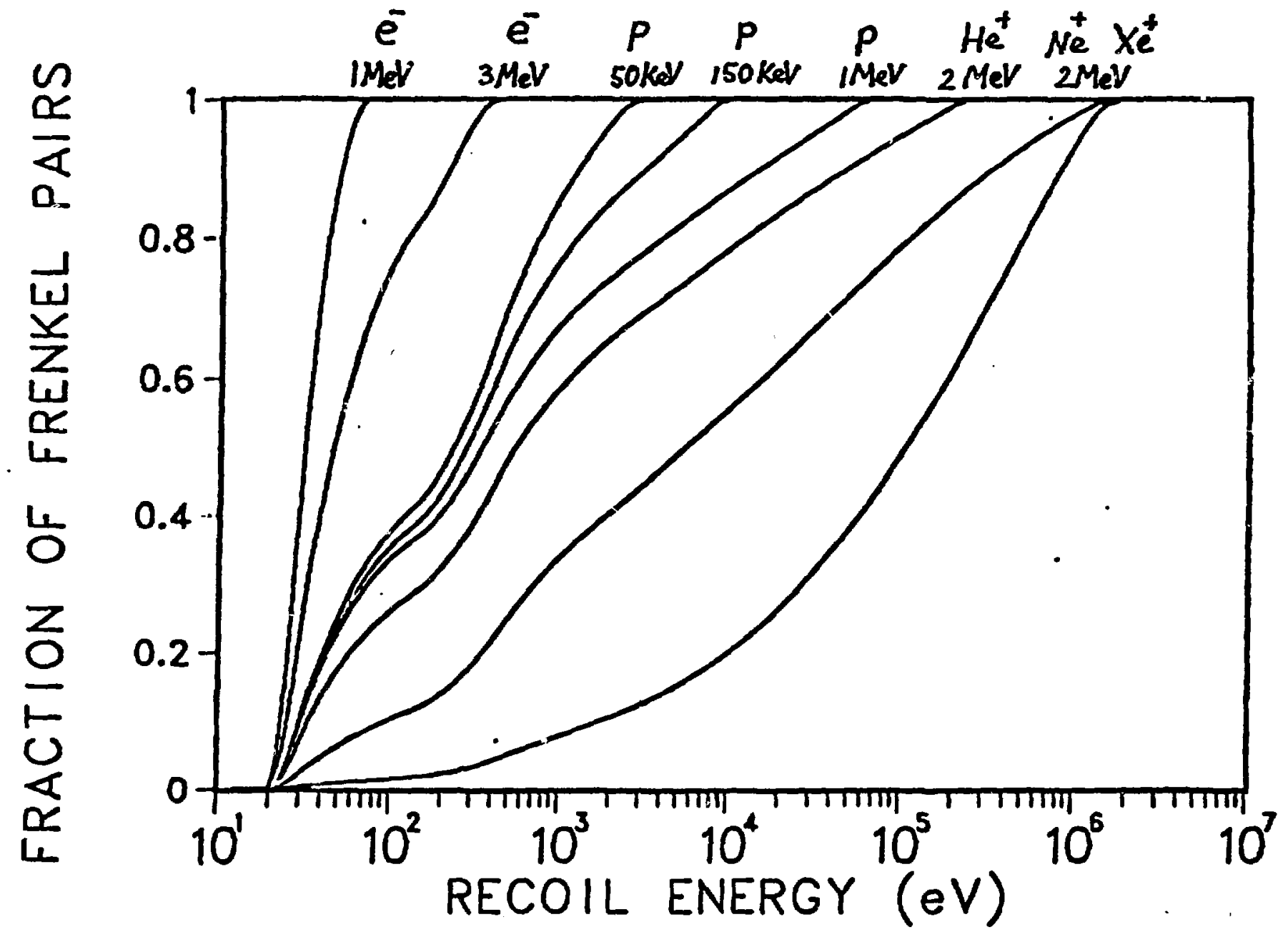

$$
\text { Fig. } 3
$$




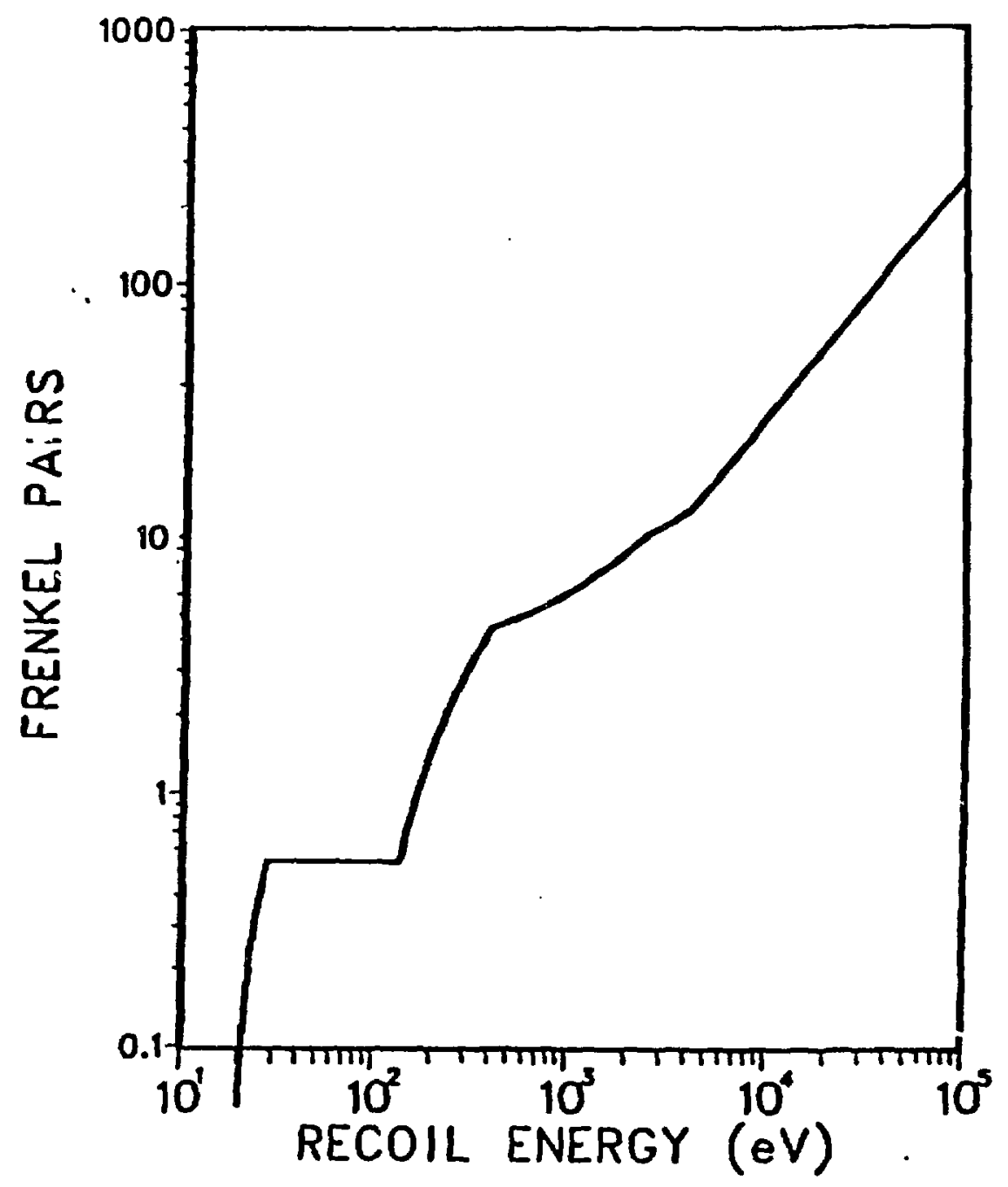

Fig. 4 
EFFICIENCY,

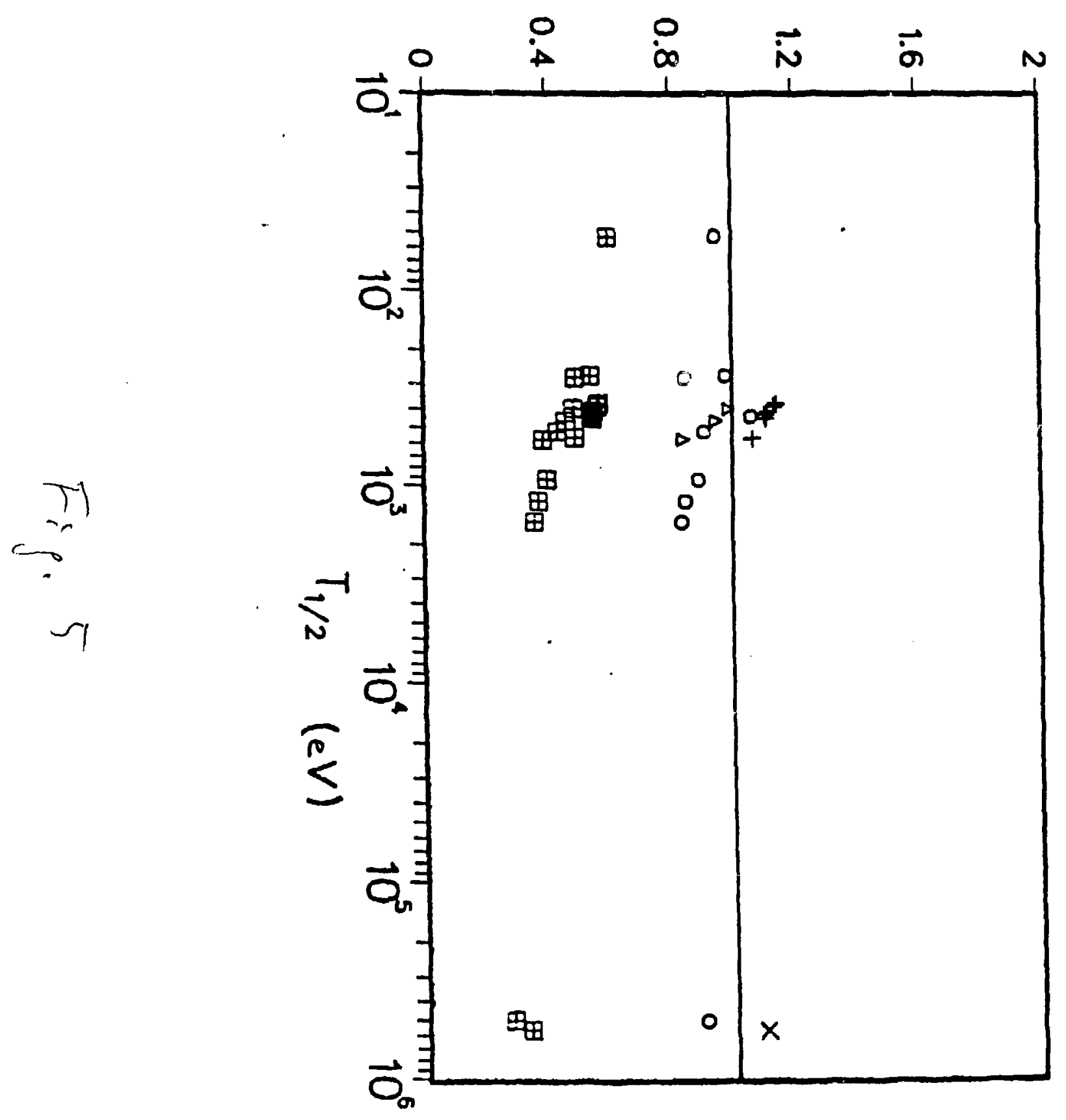

